\title{
Synthesis and Evaluation of Some Local Anti- corrosion (anti-rust) Additives for Some Petroleum Products
}

M.M. Emara, Ali M. Hassan, A. E. Salem and M. A. Ibrahim

Chemistry Department, Faculty of Science, Al Azhar University, Cairo, Egypt .

\begin{abstract}
NDER the effect of the work, lubricating oils absorb moisture from the weather and/or from the oxidation reaction on the metal surface. Therefore, anti-rust and anti-corrosion additives must be used. Rust and corrosion inhibitors, which form a barrier film on the substrate surface reducing the corrosion rate. The inhibitors also adsorb on the metal surface forming a film protecting the part from the attack of oxygen, water and other chemically active substances. The object of this study is to synthesis and evaluate of zinc octoat \& barium octoat as anti-rust, anti-corrosion additives. They were applied with oils\& greases. Synthesized additives were added with different ratio to semi synthetic cutting oil and sodium grease blends. Many trials have been carried out to obtain the optimum dose. Formulated blends were tested according to IP standard test no. 125.
\end{abstract}

Keywords: Anti-corrosion, Anti-rust, Additives, Zinc octoat, Barium octoat, Cutting oils and Greases.

The movement of two surfaces against each other is restricted due to the force that is called friction. A proper lubricant will help to minimize friction, and therefore a vital factor necessary for engines and machines to work properly. Its main function is to reduce wear and heat between two sliding surfaces and protecting the metal surface from corrosion. This is achieved by inserting the lower-viscosity material of the lubricant between the two surfaces having a relatively high-coefficient of friction. In addition, lubricants also conduct important tasks such as flush out contaminants, and absorb shocks as well as seal foams and so forth ${ }^{(1,2)}$.

A typical lubricant is comprised of more than $80 \%$ of a base-oil that together with various additives makes up the final product. The base oil predefines a whole range of property qualities such as high biodegradability, low volatility, high solvency for lubricant additives, and miscibility with other type of system fluids, among other. It has also a major influence on other qualities like oxidative stability, low temperature solidification, hydrolytic stability and viscometric properties. On the other hand, additives and/or impurities are mainly responsible for lubricity, antiwear protection, load caring capacity, corrosion prevention, 
acidity, ash content, color, foaming, demulsibility, water rejection and so forth. Base oil is mainly derived from distillation of crude petroleum and is classified as refined or synthetic. Paraffinic and napthenic oils are refined from crud oil and synthetic oils are manufactured from chemically-derived precursors ${ }^{(2-4)}$.

Grease is composed of a fluid lubricant, a thickener and additives. The function of a thickener is to constitute a kind of three-dimensional structure that will hold the fluid in place in order for it to execute its lubricating properties. The fluid can be mineral, synthetic or vegetable oil. Thickeners can be soaps and organic or inorganic soap thickeners.

The function of grease is to remain in contact with and lubricate moving surfaces without leaking out under gravity or centrifugal action, or be squeezed out under pressure. Its major practical requirement is that it retains its properties under shear at all temperatures that it is subjected to during use. At the same time, grease must be able to flow into the bearing through grease guns and from spot to spot in the lubricated machinery as needed, but must not add significantly to the power required to operate the machine, particularly at start-up.

Grease is generally used for machinery that is used intermittently or in storage for long periods. Further, grease provides good lubrication for machinery that is not easily accessible for frequent lubrication and for those that operate under extreme conditions such as high temperatures, high pressures or low speed. Grease can maintain thicker films in clearances enlarged by wear and thereby provide an opportunity to extend the life span of those equipments ${ }^{(5,6)}$.

\section{Experimental}

Two methods were used for preparation of zinc \& Barium octoat (reactant process, a double decomposition or precipitate process)

\section{Reactant process}

In this process, a metal oxide, carbonate, or hydroxide reacts with acid at temperatures up to $230^{\circ} \mathrm{C}$. Water is split out and the resulting metal soap is solubilized in a hydrocarbon solvent because the metal soaps themselves are generally hard, sticky, and difficult to grind.

$$
\begin{gathered}
\mathrm{MO}+2 \mathrm{RCOOH} \underset{\text { or }}{\longrightarrow} \mathrm{M}(\mathrm{OCOR})_{2}+\mathrm{H}_{2} \mathrm{O} \\
\mathrm{M}(\mathrm{OH})_{2}+2 \mathrm{RCOOH} \stackrel{\mathrm{M}(\mathrm{OCOR})_{2}+2 \mathrm{H}_{2} \mathrm{O}}{\longrightarrow}
\end{gathered}
$$

\section{Double decomposition (precipitate process)}

In the double decomposition reaction, an inorganic metal salt such as a sulfate, chloride, acetate, or nitrate reacts with the sodium or potassium salt of the carboxylic acid in a hot aqueous solution. The metal soap precipitate is filtered, washed, dried, and milled.

Egypt. J. Chem. 54, No. 2 (2011) 


$$
\begin{aligned}
& \mathrm{KOH}+\mathrm{RCOOH} \Longrightarrow \mathrm{RCOOK}+\mathrm{H}_{2} \mathrm{O} \\
& \text { and } \\
& 2 \mathrm{RCOOK}+\mathrm{MSO}_{4} \Longrightarrow \mathrm{M}(\mathrm{OOCR})_{2}+2 \mathrm{~K}_{2} \mathrm{SO}_{4}
\end{aligned}
$$

TABLE 1. The optimum conditions for preparation of zinc octoat according to reactant process.

\begin{tabular}{|c|c|c|c|c|}
\hline $\begin{array}{c}\text { Octoic acid: } \\
\text { ZnO }\end{array}$ & $\begin{array}{c}\text { Reaction } \\
\text { time, } \mathbf{h r}\end{array}$ & Temperature, ${ }^{\mathbf{0}} \mathbf{C}$ & Zn \% wt & Yield\% wt \\
\hline $2: 1.15$ & 1 & 110 & 17.5 & 99.7 \\
\hline
\end{tabular}

TABLE 2. The optimum conditions for preparation of octoic acid with potassium hydroxide.

\begin{tabular}{|c|c|c|}
\hline Acid/KOH ratio & Temperature, ${ }^{\mathbf{0}} \mathbf{C}$ & Reaction time, $\mathbf{h r}$ \\
\hline $1: 1$ & $90: 100$ & 1 \\
\hline
\end{tabular}

TABLE 3. The optimum conditions for preparation of potassium octoat with $\mathrm{ZnSO}_{4}$.

\begin{tabular}{|c|c|c|c|}
\hline $\begin{array}{c}\text { Potassium octoat: } \\
\mathbf{Z n S O}_{\mathbf{4}}\end{array}$ & Temperature, ${ }^{\mathbf{0}} \mathrm{C}$ & $\mathbf{Z n} \% \mathbf{w t}$ & Yield\% wt \\
\hline $2: 1.1$ & $90: 100$ & 13.6 & 96 \\
\hline
\end{tabular}

TABLE 4. The optimum conditions for preparation of barium octoat according to reactant process .

\begin{tabular}{|c|c|c|c|c|}
\hline $\begin{array}{c}\text { Acid/ } \\
\mathbf{B a}(\mathbf{O H})_{\mathbf{2}}\end{array}$ & $\begin{array}{c}\text { Reaction } \\
\text { time, } \mathbf{h r}\end{array}$ & Temperature, ${ }^{\mathbf{0}} \mathbf{C}$ & Ba \%wt & Yield\%wt \\
\hline $2: 1.15$ & 1 & 110 & 16 & 99.7 \\
\hline
\end{tabular}

TABLE 5. The optimum conditions for preparation of potassium octoat with $\mathrm{BaCl}_{2}$.

\begin{tabular}{|c|c|c|c|}
\hline $\begin{array}{c}\text { Potassium octoat: } \\
\mathbf{B a C l}_{\mathbf{2}}\end{array}$ & Temperature, ${ }^{\mathbf{0}} \mathbf{C}$ & Ba \% wt & Yield\% wt \\
\hline $2: 1$ & $90: 100$ & 15 & 99.5 \\
\hline
\end{tabular}




\section{Additives evaluation}

The two synthesized additives were blended with locally semi-synthetic cutting oil with different ratio until we obtain the optimum dose $\%$ wt and pass in both blend stability (IP 311) and emulsion stability (IP 263). Anti-corrosion properties for the blends according to IP 125 were evaluated to select the optimum dose. Also, the two synthesized additives are blended with locally sodium grease with different ratio until we obtain the optimum dose \% wt. Anticorrosion properties for the blends according to IP 112 were evaluated to select the optimum dose.

\section{Results and Discussion}

Chemical structure of zinc octoat,

$$
\begin{aligned}
\mathrm{ZnO}+2 \mathrm{RCOOH} & \Rightarrow \mathrm{Zn}(\mathrm{OCOR})_{2}+\mathrm{H}_{2} \mathrm{O} \\
\mathrm{KOH}+\mathrm{RCOOH} & \Rightarrow \mathrm{RCOOK}+\mathrm{H}_{2} \mathrm{O} \\
& \text { and } \\
2 \mathrm{RCOOk}+\mathrm{ZnSO}_{4} & \Rightarrow \mathrm{Zn}(\mathrm{OCOR})_{2}+\mathrm{K}_{2} \mathrm{SO}_{4}
\end{aligned}
$$

Chemical structure of barium octoat,

$$
\begin{gathered}
\mathrm{Ba}(\mathrm{OH})_{2}+2 \mathrm{RCOOH} \Longrightarrow \mathrm{Ba}(\mathrm{OCOR})_{2}+\mathrm{H}_{2} \mathrm{O} \\
\mathrm{KOH}+\mathrm{RCOOH} \Longrightarrow \mathrm{RCOOK}+\mathrm{H}_{2} \mathrm{O} \\
\quad \text { and } \\
2 \mathrm{RCOOK}+\mathrm{BaCl}_{2} \Longrightarrow \mathrm{Ba}(\mathrm{OCOR})_{2}+2 \mathrm{KCl}
\end{gathered}
$$

\section{Characterization of additives}

The chemical structures were confirmed via IR analysis as shown in Fig. $1,2 \& 3$

From Fig. 1- 3 spectral data observed are:

Band at wave no. $2960 \mathrm{~cm}^{-1}$ characteristic for $-\mathrm{OH}$ group

Band at wave no. $1550 \mathrm{~cm}^{-1}$ characteristic for $\mathrm{Zn}$ octoate

Band at wave no. $1380 \mathrm{~cm}^{-1}$ characteristic for $\mathrm{CH}_{3}$ group

Band at wave no. $1462 \mathrm{~cm}^{-1}$ characteristic for $\mathrm{CH}_{2}$ group

Band at wave no. $1548 \mathrm{~cm}^{-1}$ characteristic for Ba octoate

Band at wave no. $1689 \mathrm{~cm}^{-1}$ characteristic for $\mathrm{C}=\mathrm{O}$ group 


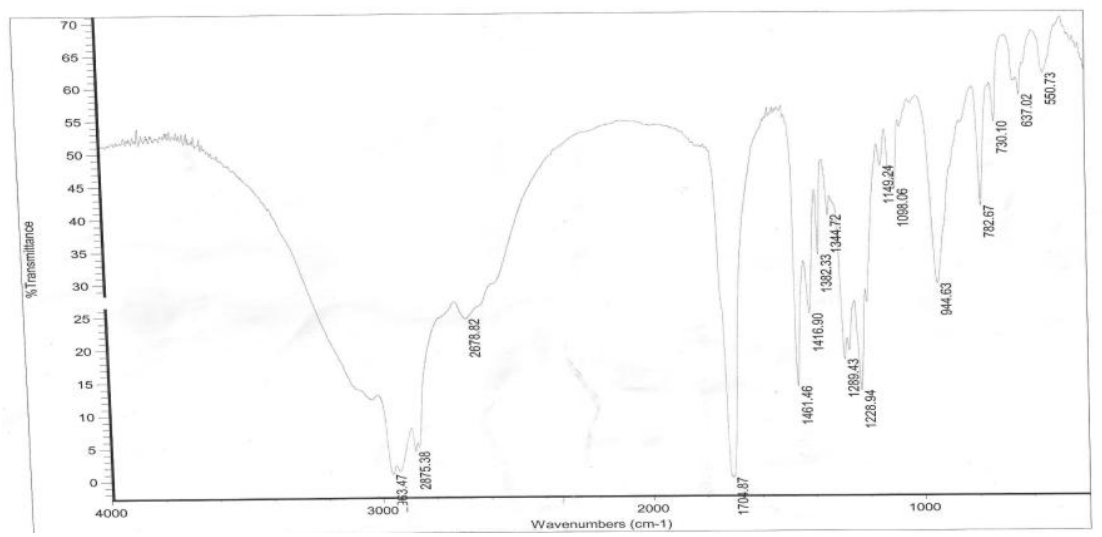

Fig. 1. IR spectral for octoic acid.

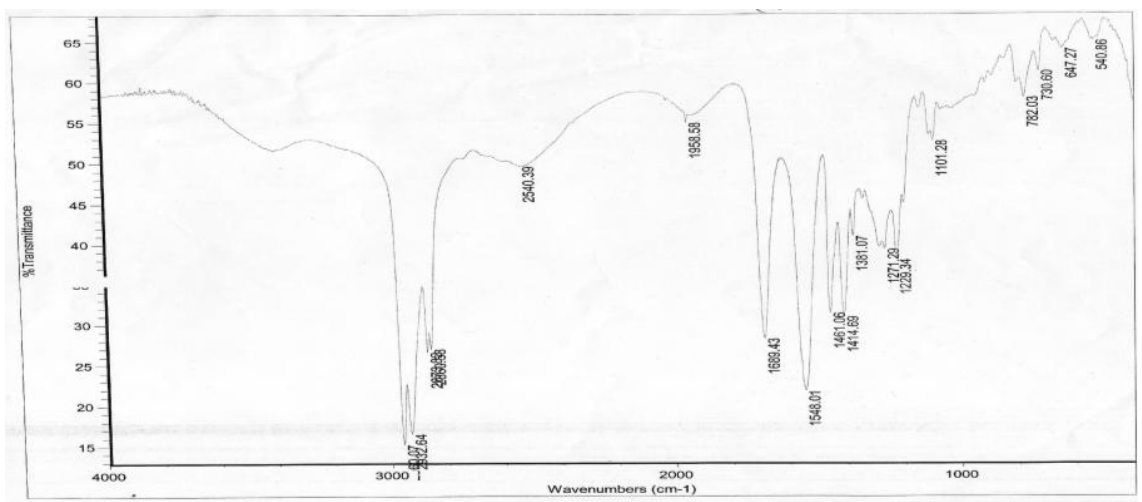

Fig. 2. IR spectral data for barium octoat.

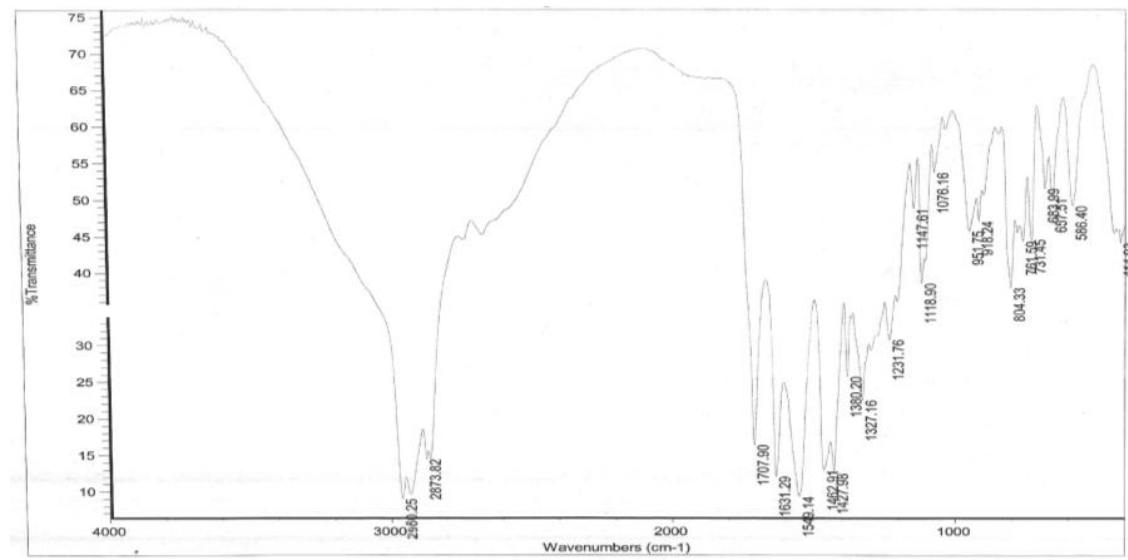

Fig. 3. IR spectral data for zinc octoat.

Egypt. J. Chem. 54, No. 2 (2011) 
TABLE 6. Cutting oil blend trials with locally zinc octoate additive.

\begin{tabular}{|l|c|c|c|c|c|c|c|c|}
\hline $\begin{array}{l}\text { Blend \% } \\
\text { wt }\end{array}$ & $\mathbf{1}$ & $\mathbf{2}$ & $\mathbf{3}$ & $\mathbf{4}$ & $\mathbf{5}$ & $\mathbf{6}$ & $\mathbf{7}$ & $\mathbf{8}$ \\
\hline Base oil & 76.8 & 76.5 & 76.2 & 76 & 75.7 & 75.5 & 75.2 & 75 \\
\hline S.N.S & 8 & 8 & 8 & 8 & 8 & 8 & 8 & 8 \\
\hline NP.E & 5 & 5 & 5 & 5 & 5 & 5 & 5 & 5 \\
\hline Oleic acid & 6.5 & 6.5 & 6.5 & 6.5 & 6.5 & 6.5 & 6.5 & 6.5 \\
\hline T.E.A & 1.5 & 1.5 & 1.5 & 1.5 & 1.5 & 1.5 & 1.5 & 1.5 \\
\hline P.E.G & 2 & 2 & 2 & 2 & 2 & 2 & 2 & 2 \\
\hline $\begin{array}{l}\text { Anti-rust } \\
\text { (Zn octoate) }\end{array}$ & 0.2 & 0.5 & 0.8 & 1 & 1.3 & 1.5 & 1.8 & 2 \\
\hline $\begin{array}{l}\text { Blend stability } \\
\text { IP 311 }\end{array}$ & pass & pass & pass & pass & pass & pass & pass & pass \\
\hline $\begin{array}{l}\text { Emulsion } \\
\text { stability }\end{array}$ & pass & pass & pass & pass & pass & pass & pass & pass \\
\hline IP125 & $3 / 3$ & $3 / 3$ & $3 / 3$ & $2 / 3$ & $1 / 3$ & $0 / 2$ & $0 / 1$ & $0 / 0$ \\
\hline
\end{tabular}

TABLE 7. Cutting oil blend trial with barium octoate additive.

\begin{tabular}{|l|c|c|c|c|c|c|c|}
\hline \multicolumn{1}{r|}{ Sample } & $\mathbf{1}$ & $\mathbf{2}$ & $\mathbf{3}$ & $\mathbf{4}$ & $\mathbf{5}$ & $\mathbf{6}$ & $\mathbf{7}$ \\
\hline Blend\% wt & 76.8 & 76.5 & 76.2 & 76 & 75.7 & 75.5 & 75.2 \\
\hline S.N.S & 8 & 8 & 8 & 8 & 8 & 8 & 8 \\
\hline NP.E & 5 & 5 & 5 & 5 & 5 & 5 & 5 \\
\hline Oleic acid & 6.5 & 6.5 & 6.5 & 6.5 & 6.5 & 6.5 & 6.5 \\
\hline T.E.A & 1.5 & 1.5 & 1.5 & 1.5 & 1.5 & 1.5 & 1.5 \\
\hline P.E.G & 2 & 2 & 2 & 2 & 2 & 2 & 2 \\
\hline $\begin{array}{l}\text { Anti-rust } \\
\text { (Ba octoate) }\end{array}$ & 0.2 & 0.5 & 0.8 & 1 & 1.3 & 1.5 & 1.8 \\
\hline $\begin{array}{l}\text { Blend stability } \\
\text { IP 311 }\end{array}$ & pass & pass & pass & pass & pass & pass & pass \\
\hline Emulsion stability & pass & pass & pass & pass & pass & pass & pass \\
\hline IP125 & $3 / 3$ & $3 / 3$ & $3 / 3$ & $2 / 3$ & $0 / 2$ & $0 / 1$ & $0 / 0$ \\
\hline
\end{tabular}


TABLE 8. Sodium greas blend trial with locally $\mathrm{Zn}$ octoate additive.

\begin{tabular}{|l|c|c|c|c|c|c|}
\hline $\begin{array}{l}\text { Sample } \\
\text { Blend\% wt }\end{array}$ & $\mathbf{1}$ & $\mathbf{2}$ & $\mathbf{3}$ & $\mathbf{4}$ & $\mathbf{5}$ & $\mathbf{6}$ \\
\hline Base oil & 86 & 85.5 & 85 & 84.5 & 84 & 83.5 \\
\hline $\begin{array}{l}\text { Stearic } \\
\text { acid }\end{array}$ & 11.15 & 11.15 & 11.15 & 11.15 & 11.15 & 11.15 \\
\hline $\mathrm{NaOH}$ & 1.85 & 1.85 & 1.85 & 1.85 & 1.85 & 1.85 \\
\hline$(\mathrm{Zn}$ octoate $)$ & 1 & 1.5 & 2 & 2.5 & 3 & 3.5 \\
\hline IP112 & black & black & black & black & black & negative \\
\hline
\end{tabular}

TABLE 9. Sodium grease blend trial with locally Ba octoate additive.

\begin{tabular}{|l|c|c|c|c|c|}
\hline Blend\% wt & $\mathbf{1}$ & $\mathbf{2}$ & $\mathbf{3}$ & $\mathbf{4}$ & $\mathbf{5}$ \\
\hline Base oil & 86 & 85.5 & 85 & 84.5 & 84 \\
\hline $\begin{array}{l}\text { Stearic } \\
\text { acid }\end{array}$ & 11.15 & 11.15 & 11.15 & 11.15 & 11.15 \\
\hline $\mathrm{NaOH}$ & 1.85 & 1.85 & 1.85 & 1.85 & 1.85 \\
\hline$($ Ba octoate $)$ & 1 & 1.5 & 2 & 2.5 & 3 \\
\hline IP112 & black & black & black & black & negative \\
\hline
\end{tabular}

\section{Conclusion}

From this study it can be possible to synthesis Zn\&Ba octoate locally. It can be used as anti-corrosion/ anti-rust additives in cutting oils and sodium grease.

\section{References}

1. US Army Corps of Engineers, Engineering and design, Lubricants and Hydraulic Fluids, Engineer Manual. 1110-2-1424, Washington, DC 20314-1000 (1999).

2.Carlsson, A.S., Production of Wax Ester in Crambe. CNAP, University of York (2006).

3.Erhan, S.Z. and Asadauskas, S., Lubricant basestocks from vegetable oils. Industrial Crops and Products, 11, 277-282 (2000) 
4 . Smith, K. W., Starr, W.C. and Chen, A new process for dewaxing lube stocks mobil lube dewaxing. API 45 Midyear Refining Meeting, May (1980).

5. Najafi, M. F. and Zambrano, L.B., Naphthenic Specialty Oils for Greases.

6. Boehringer, R. H., Grease. In : ASM Handbook, Friction, Lubrication and Wear Technology, Volume 18, ASM International, U.S.A., p123 (1992) .

(Received 1/8/2011;

accepted 16/8/2011)

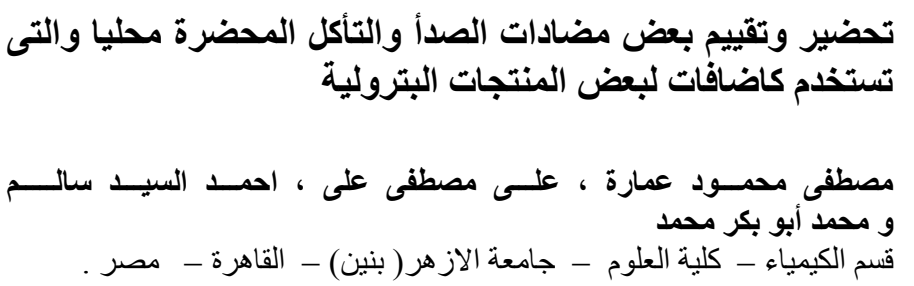

نتيجة لتأثير الثغل وامتصاص زيوت النزييت الرطوبة من الجو وايضا تفاعل

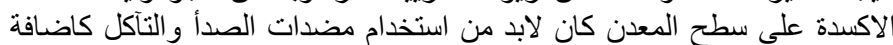

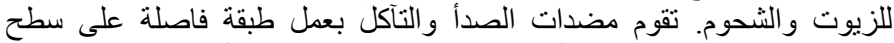

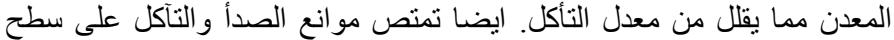

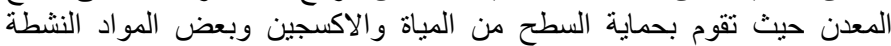

كميائيا.

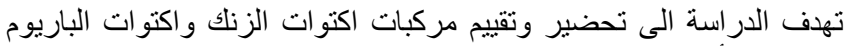

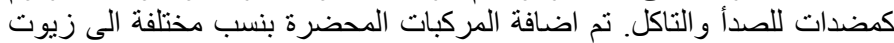

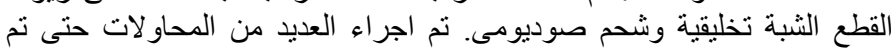

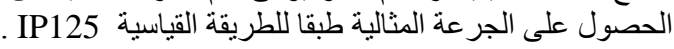

Egypt. J. Chem. 54, No. 2 (2011) 